\title{
Filtering and analyzing normal and abnormal electromyogram signals
}

\author{
S. Elouaham ${ }^{1}$, A. Dliou' ${ }^{2}$ M. Laaboubi ${ }^{3}$, R.Latif ${ }^{4}$, N. Elkamoun ${ }^{5}$, H. Zougagh ${ }^{6}$ \\ ${ }_{1,5}$ LSTIC, Faculty of sciences, ChouaibDoukkali University, Morocco \\ ${ }^{1,2,3,4}$ LISTI, National School of Applied Sciences, IbnZohr University, Morocco \\ ${ }^{6}$ Faculty of sciences and techniques, University BeniMellal, Morocco
}

\section{Article Info}

Article history:

Received Jan 27, 2020

Revised Apr 1, 2020

Accepted Apr 15, 2020

\section{Keywords:}

AOK

CEEMDAN

Choi-Williams

EMG

MAUPs

\begin{abstract}
The electromyogram (EMG) is an important measurement to assess the health of muscles and the nerve cells that control them. The appearance of noise in electromyography (EMG) signals may unquestionably minimize the efficiency of the analysis of the signal. The denoising techniques are inevitable for minimizing noise affecting the EMG signals; these methods are Complete Ensemble Empirical Mode Decompositions with Adaptive Noise (CEEMDAN) and the Ensemble Empirical Mode Decomposition (EEMD). After that, we analyze these signals by time-frequency techniques as Adaptive Optimal Kernel (AOK) and Choi-Williams. Firstly, the obtained results illustrate the effectiveness of the CEEMDAN that permits reducing noise that interferes with normal and abnormal EMG signals with higher resolution than other techniques used as EEMD. Secondly, they show that the AOK technique is adapted to the detection and classification of these types of normal and abnormal EMG signals by the good localization of the Motor Unit Action Potentials (MUAPs) in the time-frequency plan. This paper shows the efficiency of the combination of the AOK and CEEMDAN techniques in analyzing the EMG signals.
\end{abstract}

Copyright (C) 2020 Institute of Advanced Engineering and Science. All rights reserved.

\section{Corresponding Author:}

Elouaham Samir,

Faculty of sciences.

ChouaibDoukkali University, El Jadida, Morocco.

Email: elouahamsamir@gmail.com

\section{INTRODUCTION}

The signal electromyogram (EMG) is a clinical test commonly used to evaluate the function of muscles and nerves [1]. The analysis of EMG signals is used to help the doctors at rapid diagnosis, to detect disorders such as muscular dystrophy and neuropathies. The studies of nerve conduction, which measure to what extent and at what speed are the pulses from the nerves of conduct, are often conducted in collaboration with studies of signals [1-7]. The objective of this paper is to reveal muscle fatigue by the analysis of the EMG signals. The EMG signal is composed of waves called Motor Unit Action Potential (MUAP) [1-7]. The factors of the classification of normal and abnormal signals are dependent on the form, the amplitude and the frequency contents of the MUAPs. The Detecting of the MUAPs reflect the electrical activity of a single anatomical motor unit. It represents the potential of composite action of these muscle fibers on the inside of the recording range of the electrode. The proper EMG signal is a requirement for a suitable examination. During the recording of the EMG signals, the natural noises can be contaminated with the original signals. Because of noise that interferes with the EMG signal, the loss of a portion of the surface EMG signal can contribute to the underestimation of EMG activity. The main noise sources are the artifact related to movement and electrical noise. These noises disrupt the useful information visualization of the signal. To overcome this problem, several techniques are used for filtering biomedical signals such as Empirical Mode Decomposition (EMD) and Ensemble Empirical Mode Decompositions (EEMD) [8-11]. 
These methods are tested on EMG signals available in the database Physionet [12]. The disadvantages of EMD are the appearance of mode-mixing effect in signal restoration and also this method can't separate the noises that have some oscillations interfering with normal and abnormal EMG signals. The EEMD technique and the complete ensemble empirical mode decomposition (CEEMDAN) are presented in this paper for overcoming the drawbacks of the EMD method [9-10]. The EMG signals are non-stationary and non-linear. The origin of the non-stationarity of EMG signals can also be linked to variables such as changes in the level of muscle contraction, moving the electrodes, changes in the shape of MUAP over time, and changes in the inter-peak interval MUAPs [13-14]. The EMG signals have a lot of information that permits to reveal of the anomaly of each case. Analyzing the EMG signals is often need for methods that aid the doctor to give all information existing in biomedical signals. Traditional methods are often used to analyze EMG. These methods don't give all the information about the EMG signal because of the non-stationarite and non-linearity of this signal. In the first method classic used, MUAPs are detected in the time domain. Despite the fact the study of MUAPs in the temporal domain affords a relatively simple method to identify the form of MUAP when it arises, it does not give any information about the frequency content. In the second method classic, the Fourier transform gives the frequency content of the signal without giving information about the time of the apparition of the energy of the MUAP [15]. For surpassing these problemes of these classics methods the time-frequency techniques are used [16-19]. Among the goals of this paper are the presents the methods that able to associate the advantages of the temporal and frequency domains and give the frequency contents in the time-frequency plan. This frequency contents give all information that explain the anomaly. In this work, we illustrate the extraction of the contents of EMG signals by using the timefrequency techniques [20-28]. This extraction is dependent on the detection of the MUAPs. The Detection of the MUAPs is a fundamental step that provides relevant information on the neuromuscular system. We will compare visually how the activity of MUAPs appearing on the Adaptive Optimal Kernel (AOK) technique with the visualizations provided by the Choi-Williams method [20-28]. Since such comparisons of timefrequency techniques to analyze MUAPs do not exist that much, results and ideas presented in this work can be helpful in any investigation which is concerned with the future selection of an appropriate technique for analysis of muscle contraction.

The paper is formulated as follows: Section 2 gives the research method; this section describes the denoising and the time-frequency methods, and provides the normal and abnormal electromyogram signals. The results and analysis of the denoising techniques and time-frequency ones are elaborated on in Section 3. Finally, conclusions are provided in Section 4.

\section{RESEARCH METHOD}

\subsection{Denoising techniques}

\subsubsection{Empirical mode decomposition (EMD)}

The EMD technique is defined in [9]. The goal of this technique is the decomposed the signal into a set of AM-FM components [9-11]. Despite the fact that this technique does not use any hypothesis or mathematical definitions, it gives good results in different fields like biomedical [9-11]. The EMD technique decomposes the non-stationary signal into a sum of functions namedIntrinsic Mode Functions $\operatorname{IMF}_{\mathrm{J}}(\mathrm{t})$. The signal $\mathrm{x}(\mathrm{t})$ is given by:

$$
x(t)=\sum_{j=1}^{k} d_{j}(t)+s(t)
$$

Withs $(t)$ is the residue of the signal. The $d$ is IMF.

The algorithm of the EMD technique is given as follows:

a. Calculation of the average envelope ( the average of the minimum envelope and the maximum envelope)

$$
m(t)=\left(e_{\max }(t)+e_{\min }(t)\right) / 2
$$

b. a local detail is obtained as follow :

$$
d(t)=x(t)-m(t)
$$

c. The expression (1) gives the iteration. 
The decomposition distributed the signal as follows; the first IMF contains the high frequencies, which that imply the following terms of decreasing frequency until the last IMF contains the low frequency.

\subsubsection{Ensemble Empirical Mode Decomposition (EEMD)}

The Ensemble Empirical Mode Decomposition (EEMD) method is suggested to overcome the problem of the mode-mixing that appearing in EMD method [10]. By reiteration, the operation of decomposition the EMD gives the true IMF.The EEMD algorithm is given by the steps following:

Step 1:we add a predefined noise on the analyzed signal.

Step 2:we apply the EMD technique on the resulted signal.

Step 3: signal decomposition is done by the white noise of fixed amplitude.

Step 4: decomposition final is the means ensemble means of the decomposition results.

As the finite number of (IMFs) and a residue, the signal $\mathrm{x}(\mathrm{k})$ is decomposed.

$$
x(k)=\sum_{i=1}^{n} c_{i}+r
$$

With $\mathrm{n}$ is the IMFs number, ${ }^{\prime} c_{i}$ is the ith IMF that corresponding IMF ensemble mean resulted given by all of operations of the decomposition and $r$ is the residues mean given by all operations of the decomposition.

\subsubsection{Complete ensemble empirical mode decomposition with adaptive noise (CEEMDAN)}

In one hand, using EEMD overcomes the EMD mode mixing problem, but in other hand this technique presents a problem. The number of iterations required in EEMD process is higher, added to the residual noise remaining in the reconstructed signal. The new version of the EEMD technique produces a correct restoration of a non-stationary signal and provides a good separation of IMF with low calculation cost. The $\overline{I M F}_{1}(n)$ is given by applied the EEMD technique. Then count first EMD mode over an ensemble of $r_{1}(n)$ plus different realizations of a given noise obtaining $\overline{I M F}_{2}(n)$ by averaging. Here Ej [.] operator provide jth mode obtained by EMD. wi is the white noise[11].

The CEEMDAN algorithm is obtained as follows:

Step 1: the first IMF is obtained by decomposing the signal $x(n)+w_{0} \varepsilon^{i}(n)$ by using:

$$
\overline{I M F}_{1}(n)=\frac{1}{I} \sum_{i=1}^{l} \overline{I M F_{k}^{i}}(n)
$$

With $w_{0}$ is amplitude of the noise, and $\varepsilon(t)$ is the white noise with unit variance.

Step 2: Calculate the difference signal:

$$
r_{1}(n)=x(n)-\overline{I M F}_{1}(n)
$$

Step 3: The second mode is obtained by decomposing the $r_{1}(n)+w_{1} E_{1}\left(\varepsilon^{i}(n)\right)$ by using:

$$
\overline{I M F}_{2}(n)=\frac{1}{I} \sum_{i=1}^{l} E_{1}\left(r_{1}(n)+w_{1} E_{1}\left(\varepsilon^{i}(n)\right)\right)
$$

For $\mathrm{k}=2, \mathrm{~K}$, compute the $\mathrm{k}$-th residue and obtain the first mode. Define the $(\mathrm{k}+1)$-th mode as follows:

$$
\overline{I M F}_{k+1}(n)=\frac{1}{I} \sum_{i=1}^{l} E_{1}\left(r_{k}(n)+w_{k} E_{k}\left(\varepsilon^{i}(n)\right)\right)
$$

where E1 is a function to extract the $\mathrm{j}$-th IMF decomposed by EMD

Step 4: We complete this operation until residue no longer achievable. Final residue

$$
R(n)=x(n)-\sum_{k=1}^{k} \overline{I M F}_{k}
$$


The original signal is given as follows:

$$
x(n)=R(n)+\sum_{k=1}^{k} \overline{I M F}_{k}
$$

These methods are applied on MIT-BIH Arrhythmia database [12].

\subsection{Time-frequency techniques}

The time-frequency techniques are applied to non-stationary signals, these techniques are in great demand in the field which requires representing the frequency spectrum over time to extract information useful that aid the give a good diagnosis. Among the methods chosen in this study, we chose the techniques of Choi-Williams and AOK [20-23].

\subsubsection{Choi-Williams distribution (CWD)}

The among the goals of the Choi-Williams technique $\operatorname{CWD}(t, f)$ is the resolution the non-stationary signal and the reduction of cross-terms [22-23].

$$
\begin{aligned}
& C W D_{x}(t, f)=\frac{1}{4 \pi^{2}} \int_{-\infty}^{\infty} \int_{-\infty}^{+\infty} \int_{-\infty}^{-j \theta t-j \tau \omega+j \theta u} \phi(\theta, \tau) A_{u} d u d \tau d \theta \\
& \text { Where }_{A_{u}}=x\left(u+\frac{\tau}{2}\right) x^{*}\left(u-\frac{\tau}{2}\right) \\
& \text { And } \phi(\theta, \tau)=e^{\frac{\theta^{2} \tau^{2}}{\sigma}}
\end{aligned}
$$

\subsubsection{Adaptive optimal kernel (AOK)}

The Adaptive Optimal kernel time-frequency technique is represented by equation [20-21].

$$
A O K_{X}(t, f)=\frac{1}{4 \pi^{2}} \int_{-\infty}^{\infty} \iint_{-\infty}^{+\infty} \exp ^{-j \theta t-j \tau \omega+j \theta u} \phi(\theta, \tau) A_{u} d u d \tau d \theta
$$

Where $e \frac{\left(\theta^{2}+\tau^{2}\right)}{2 \sigma^{2}}(\psi)$

Where $\phi(\theta, \tau)$ is the kernel. $x^{*}(\mathrm{t})$ is the complex associate of the real signal $\mathrm{x}(\mathrm{t})$ of the real signal.

\subsection{Biomedical signals}

Electromyogram signals permit to give the human muscle states. EMG signals are used in the medical domain for analyzing the human if he has a problemof the contraction of the muscles. During this contraction, the electrical currents are generated for giving the MUAPs in the time domain. The Figure 1 shows an example of a normal EMG signal [12]. The Figure 2 gives the old man with neuropathy [12]. And the Figure 3 represents and man with myopathy. These signals are obtained in the database Physionet [12].

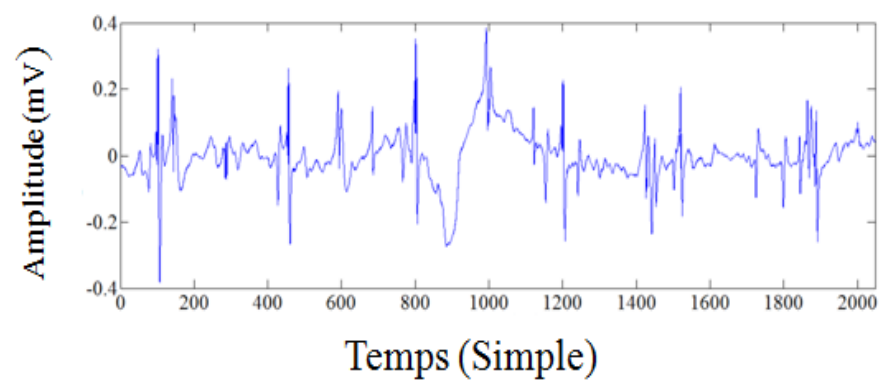

Figure 1. Normal EMG signal 


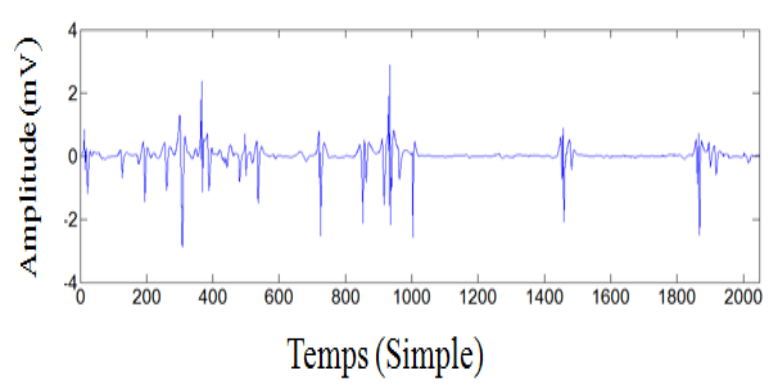

Figure 2. Abnormal EMG signal: neuropathy

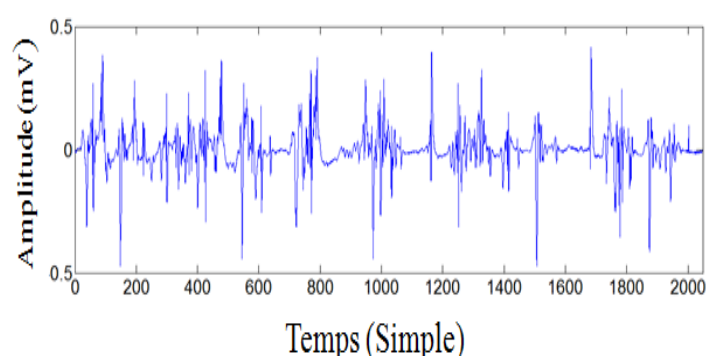

Figure 3. Abnormal EMG signal: Myopathy

\section{RESULTS AND ANALYSIS}

The results of the methods used in this research are given through by applying these techniques to the normal, myopathy and neuropathy EMG signals. The first part of this section gives the results of the CEEMDAN and EEMD techniques; the second section gives the results of the AOK and ChoiWiliams Distributions.

\subsection{Denoising technique}

The major problems of the biomedical signals are the noises that interfered with EMG signals. These artifacts provide bad information to a doctor.To overcome these problems the denoising methods are used for eliminating the artifacts that contaminated with EMG signals. The denoising techniques EEMD and CEEMDAN are applied to the normal, neuropathy and myopathy EMG signals in this part.

The performance of the CEEMDAN technique is compared with EEMD technique.The metrics used in this research are Mean Square Error (MSE) and Percent Root Mean Square Difference (PRD). These metrics are given as follows:

$$
\begin{aligned}
& \text { MSE }=\frac{1}{N} \sum_{n=1}^{N}(x(n)-\bar{x}(n))^{2} \\
& P R D=\sqrt{\frac{\sum_{n=1}^{N}(x(n)-\bar{x}(n))^{2}}{\sum_{n=1}^{N} x^{2}(n)}} * 100
\end{aligned}
$$

With $\mathrm{x}(\mathrm{n})$ is the original EMG signal, $\bar{x}(n)$ is theEMG signal obtained reconstruction after using the denoising technique and $\mathrm{N}$ is the number of point used.

The evaluation of these techniques is based on the calculation of MSE and PRD parameters which have already been mentioned. Table 1 and Table 2 and the Figure 4 and Figure 5 present the comparison of results of the denoising techniques; these tables and figures present the comparative results for two abnormal signals (myopathy and neuropathy) and normal EMG signal.The noise added to the EMG signals is natural noise signal [12]. The EMG signals used are normal presented by c: Healthy and abnormals presented by a: Neuropathy and b: Myopathy.

Table 1. The results of MSE

\begin{tabular}{ccc}
\hline Signals & CEEMDAN & EEMD \\
\hline $\mathrm{a}$ & 0.0557 & 0.0602 \\
$\mathrm{~b}$ & 0.0284 & 0.0317 \\
$\mathrm{c}$ & 0.0307 & 0.0308 \\
\hline
\end{tabular}

Table 2. The results of PRD

\begin{tabular}{ccr}
\hline Signals & CEEMDAN & \multicolumn{1}{c}{ EEMD } \\
\hline $\mathrm{a}$ & 65.013 & 67.561 \\
$\mathrm{~b}$ & 191.219 & 202.886 \\
$\mathrm{c}$ & 210.173 & 210.621 \\
\hline
\end{tabular}

From the results of the tables, the CEEMDAN technique gives the low values of MSE $(a=0.0557$, $b=0.0284$ and $c=0.0307)$ and PRD $(a=65.013, b=191.219$ and $c=210.173)$ as compared with the other technique EEMDMSE(a=0.0602, $\mathrm{b}=0.0317$ and $\mathrm{c}=0.0308)$ and PRD $(\mathrm{a}=67.561, \mathrm{~b}=202.886$ and $\mathrm{c}=210.621)$. These results show that the EEMD techniques fail to improve signal qualityowing to the interaction between signal and noise and the major problem of white noise is not totally eliminated [11]. The results obtained in this section are better than the results given in our study [28]. The obtained results show the effeteness of the CEEMDAN technique during the restoration of the EMG signals without noise. 


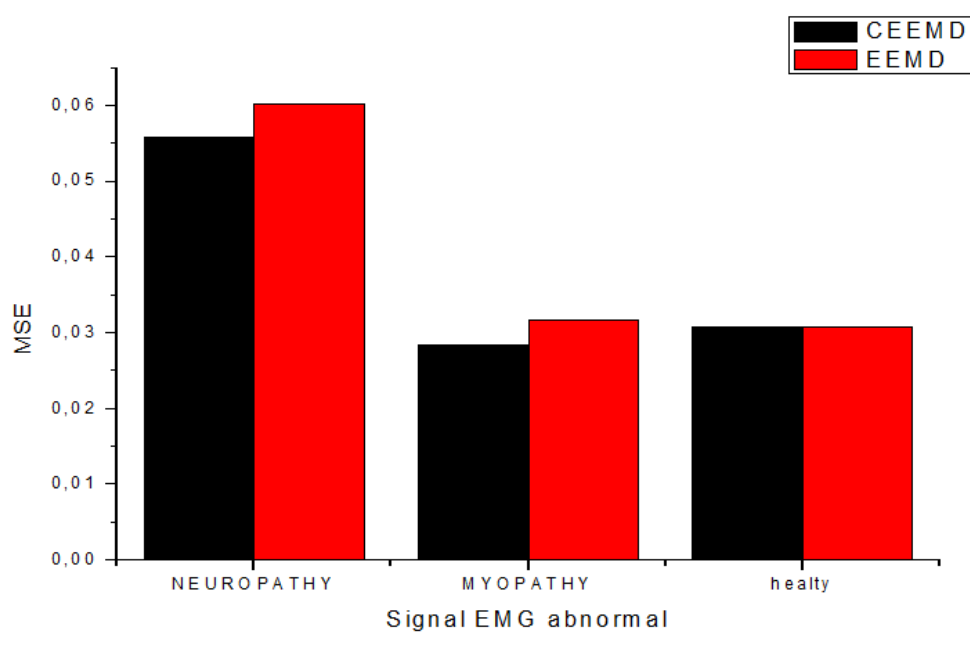

Figure 4. Results of the MSE

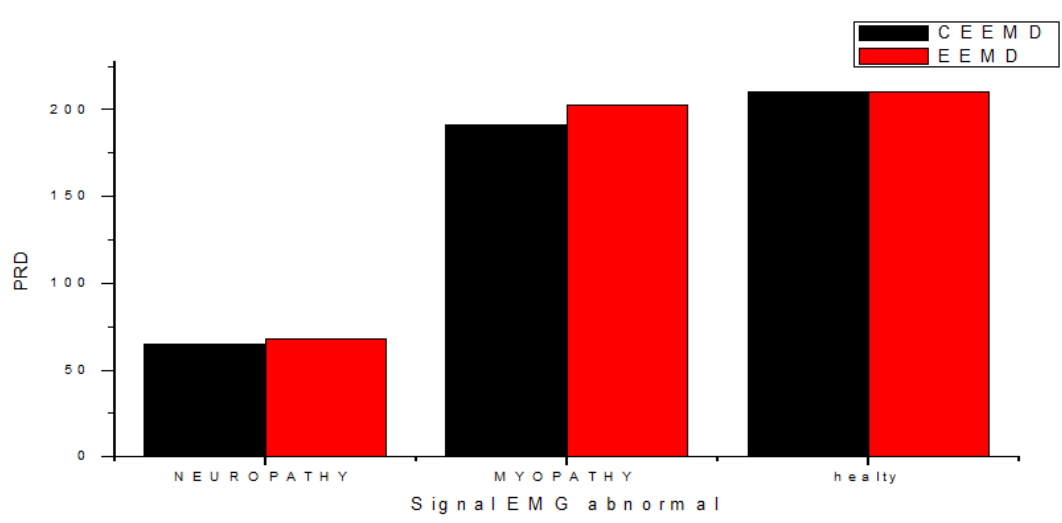

Figure 5. Results of PRD

\subsection{Time-frequencyanalysis}

The traditional methods (temporal and frequency) can not give information about the time and frequency simultaneously. To surpass these disadvantages, the time-frequency methods are used for analyzing the EMG signals. We applied the time-frequency techniques mentioned previously on non-linear and non-stationary signals, namely the electromyogram (EMG). The time-frequency methods demonstrate the distribution of the energy on the time-frequency plan. This energy provides important information about signal studying. The Choi-Williams time-frequency method has a good resolution than the other nonparametric methods depending on the variance calculated and images obtained in our studies of the results obtained in our laboratory [23].This study presents the comparison of the Choi-Williams and AOK methods. The following figures show the results obtained by the AOK and Choi-Williams method. The Figure 6(c), Figure7(c), and Figure8(c) present the time-frequency images obtained by the AOK technique and the Figure6(b), Figure7(b), and Figure8(b) present the images obtained by the Choi-Williams method. The normal and abnormal EMG signals are presented in Figure6(a), Figure7(a), and Figure8(a). In the EMG signals, the MUAPs detection is a preprocessing step used in the decomposition of EMG signals, that objective to obtain the information of the MUAPs related to the time of presence and morphology of the latter. This information makes it easier for the experts to determine proper models, which are MUAPs from the EMG signal, which are used for the analysis of neurological disarrangement. From these timefrequency images; we found that the AOK technique provides a good localization of the MUAPs in the timefrequency plan. The obtained results by AOK time-frequency proves the concentration of energy and the change of frequencies are clearly interpreted in time. This is against the Choi-Williams technique which makes visualization of the MUAPs activities extremely difficult because of the existence of interference items (cross-terms) in the time-frequency plan. These cross-terms are shown in the time-frequency images Figure6(b), Figure7(b), and Figure8(b). The resolution proposed by the AOK technique is clear in 
Figure 6(c), Figure7(c), and Figure8(c) in the time-frequency plan compared to the non-parametric technique by Choi-Williams. Notice that a disruption was presented just after the display of each MUAP. This anomaly can't identify in the time domain. The image of this signal in the time-frequency domain exposes the time the MUAPs occur and also the moment when this discontinuity appears. The MUAPs activity can be clearly seen on the time-frequency images by high levels of energy that are in synchronization with MUAPs occurrence in the time domain. The obtained results of the images have physical content; especially they perform the energy production from the exchange of sodium and potassium ions in muscle fibers over the detected MUAPs.

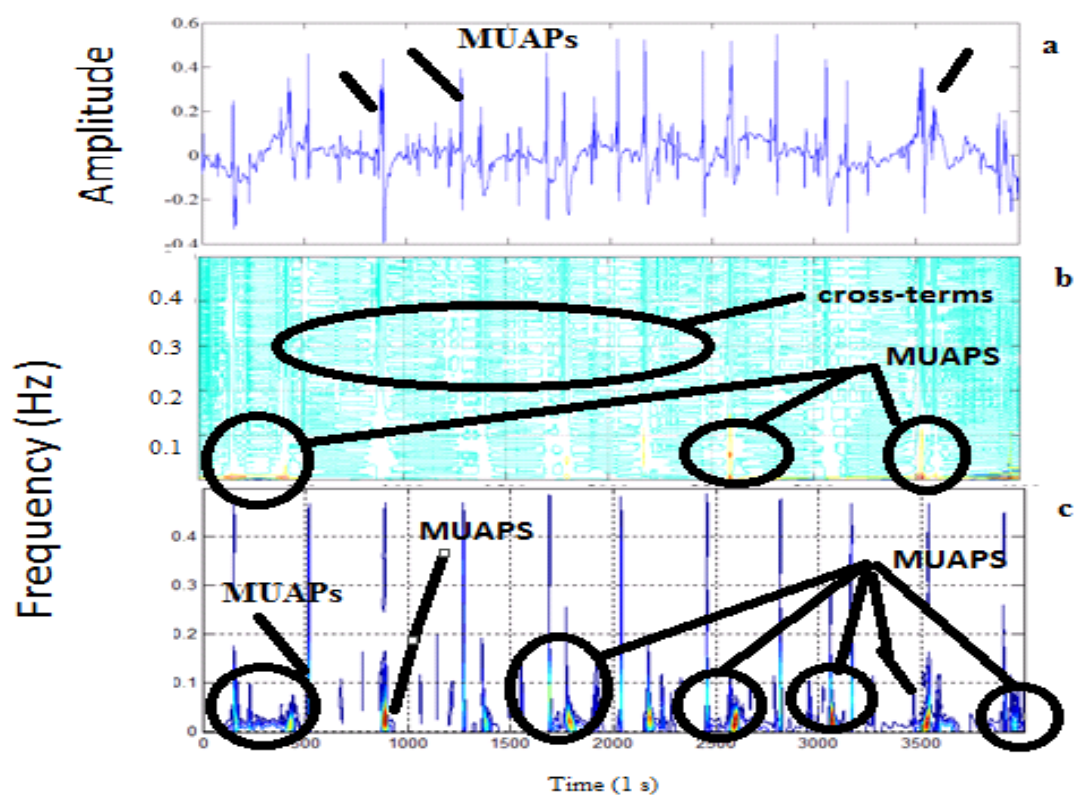

Figure 6. Time-frequency images of normal signal EMG: (a) EMG signal normal, (b) Choi-Williams, (c) $\mathrm{AOK}$

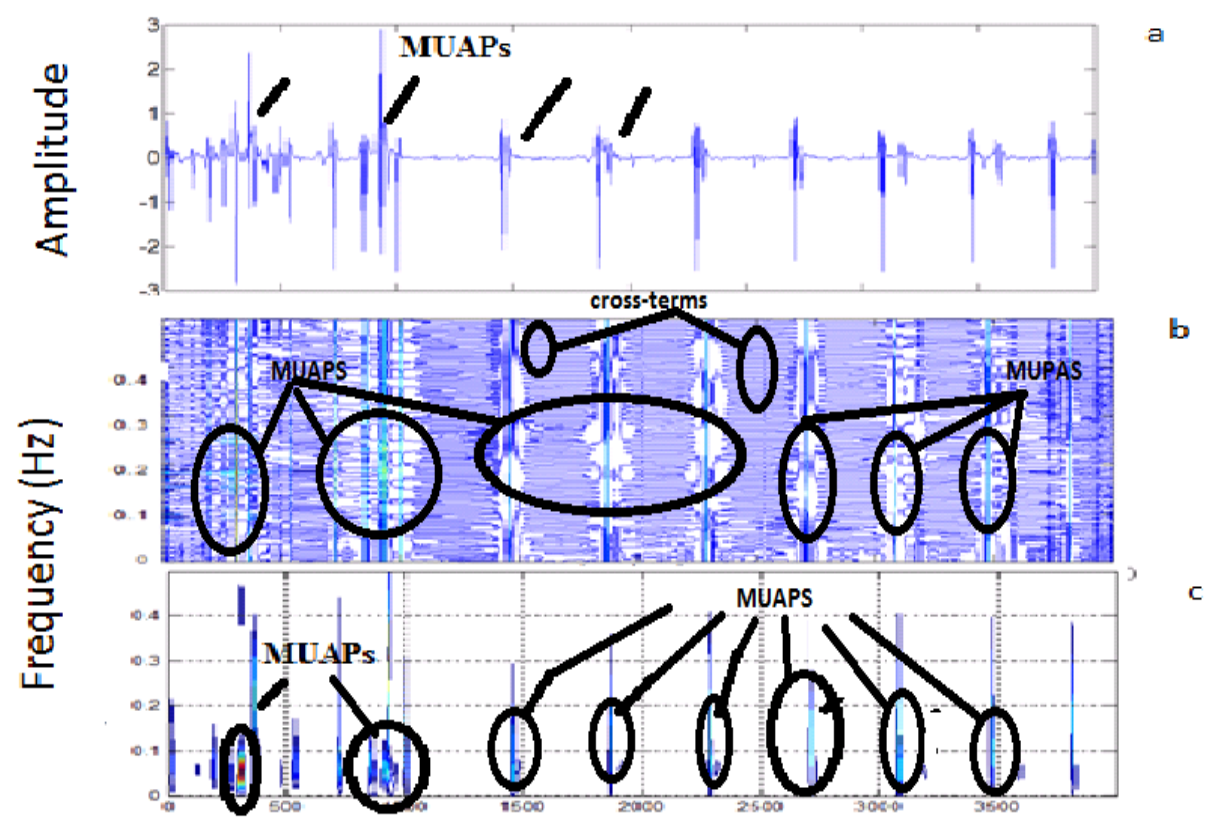

Figure 7. Time-frequency images of abnormal signal EMG: (a) EMG signal abnormal (neuropathy), (b) Choi-Williams, (c) AOK 


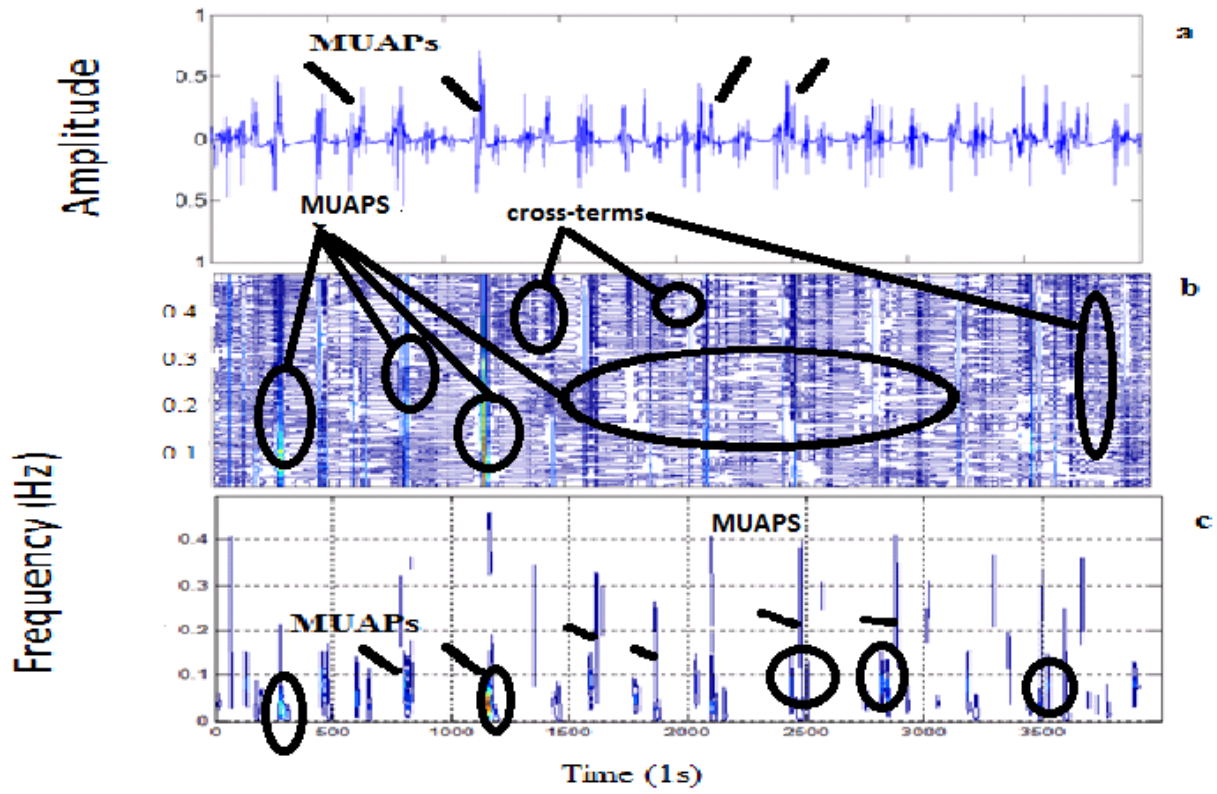

Figure 8. Time-frequency images of abnormal signal EMG: (a) EMG signal abnormal (myopathy), (b) Choi-Williams, (c) AOK

From the results, we can deduce some important characteristics of the normal, myopathy and neuropathy EMG signals by the explanation of the images obtained by the AOK technique such as the distance between MUAPs and frequency interval of the high and low energy.The goal of this section proves that the technique of AOK gives a good visualization and localization of MUAPs activities on the time-frequency plan. The MUAPs activities presented in the time-frequency plan are very important for detecting the abnormalities of each signal; that heps the expert to determine the type of anomaly signal.

\section{CONCLUSION}

In this paper, we examined the advantage of combining the CEEMDAN and the AOK techniques for analyzing the normal, neuropathy and myopathy EMG signals. The first results in this study show the effectiveness and robustness of the CEEMDAN technique in order to minimize the artifacts. The second results concerning the AOK time-frequency show a good solution for revealing all MUAPs with high resolution in order to analyze EMG signals. Time-frequency methods feature were used for the person's activity. From this analysis, we concluded that the CEEMDAN and AOK techniques are very suitable for the conservation and extraction of the characteristics of EMG signals.

\section{REFERENCES}

[1] Reaz MBI, Hussain MS and Mohd-Yasin F. "Techniques of EMG signal analysis: Detection processing, classification and applications,"Biol. Proced. Online, vol. 8, no. 1, pp. 11-35, 2006.

[2] F. Gaetani, R. de Fazio, G. A. Zappatore, P. Visconti,"A prosthetic limb managed by sensors-based electronic system: Experimental results on amputees,"Bulletin of Electrical Engineering and Informatics (BEEI), vol. 9, no. 2, pp. 514-524, 2020.

[3] M. Azlan Abu, et al., "Classification of EMG signal for multiple hand gestures based on neural network," in Indonesian Journal of Electrical Engineering and Computer Science (IJEECS), vol. 17, no. 1, pp. 256-263, 2020

[4] A. M. Najlallyana, et al., "Analysis of surface electromyography for hand gesture classification,"Indonesian Journal of Electrical Engineering and Computer Science (IJEECS), vol. 15, no. 3, pp. 1366-1373,2019.

[5] M. R. Mohamad Ismail, et al., "Hand motion pattern recognition analysis of forearm muscle using MMG signals," Bulletin of Electrical Engineering and Informatics (BEEI), vol. 8, no. 2, pp. 533-540,2019.

[6] C. Li, et al., "The signal processing and identification of upper limb motion based on sEMG,"Wireless Personal Communications, vol. 103, no. 1, pp. 887-896, 2018.

[7] S. Negi, et al, "Feature Extraction and Classification for EMG Signals using Linear Discriminant Analysis," 2016 2nd International Conference on Advances in Computing, Communication, \& Automation (ICACCA) (Fall), Bareilly, pp. 1-6,2016. 
[8] A.O. Andrade, S. Nasuto, P. Kyberd, "EMG signal filtering based on empirical mode decomposition," Biomedical Signal Processing and Control, vol. 1, no. 1, pp:44-55, 2006.

[9] G. Rilling, P. Flandrin, P. Goncalves, "On empirical mode decomposition and its algorithms,"IEEE-EURASIP Workshop on Nonlinear Signal and Image Processing, Grado-Trieste, Italy, vol. 3, no. 3, pp. 8-13, 2003.

[10] He, X.; Goubran, R.A.; Liu, X.P. "Ensemble Empirical Mode Decomposition and adaptive filtering for ECG signal enhancement," In Proceedings of 2012 IEEE International Symposium on Medical Measurements and Applications (MeMeA), Budapest, Hungary, pp. 1-5, 2012.

[11] Torres, M.E.,Colominas, M.A.; Schlotthauer, G.; Flandrin, P.,"A complete ensemble empirical mode decomposition with adaptive noise," In Proceedings of 2011 IEEE International Conference on Acoustics, Speech and Signal (ICASSP), Prague, Czech; pp. 4144-4147, 2011.

[12] Physiobank, Physionet, "Physiologic signal archives for biomedical research," viewed August2005.

[13] A.O. Andrade, S.J. Nasuto, P. Kyberd, "Extraction of motor unit action potentials from electromyographic signals through generative topographic mapping,"Journal of the Franklin Institute, vol. 344, no. (3-4), pp. 154-179, 2007.

[14] AkashKumarBhoi, et al., "Evaluation of frequency domain features for myopathic EMG signals in Mat Lab," Int. Journal of Engineering Research and Applications, vol. 3, no. 5, pp.622-627, 2013.

[15] J. Too, et al., "Featureless EMG pattern recognition based on convolutional neural network," in Indonesian Journal of Electrical Engineering and Computer Science(IJEECS), vol. 14, no. 3, pp. 1291-1297, 2019.

[16] Michele G., Sello S., Carboncini MC., Rossi B., Strambi S. "Cross-correlation time-frequency analysis for multiple EMG signals in Parkinson's disease: A wavelet approach,"Medical Engineering \& Physics, vol, 25, no. 5, pp. 361-369, 2003.

[17] P. Bonato, S. H. Roy, M. Knaflitz, and C. J. De Luca, "Time-frequency parameters of the surface myoelectric signal for assessing muscle fatigue during cyclic dynamic contractions," IEEE Transactions on Biomedical Engineering, vol. 48, no. 7, pp. 745-755, 2001.

[18] E. Merlo, M. Pozzo,G. Antonutto,P. E.di Prampero, R. Merletti, D. Farina "Time-frequency analysis and estimation of muscle fiber conduction velocity from surface EMG signals during explosive dynamic contractions" Journal of Neuroscience Methods Volume 142, Issue 2, Pages 267-274, 2005.

[19] R. Constable, R. J. Thornhill,"Using the discrete wavelet transform for time-frequency analysis of the surface EMG signal,"Biomedical Sciences Instrumentation, vol. 29, pp. 121-127, 1993.

[20] D. L. Jones and R. G. Baraniuk, "An adaptive optimal kernel time-frequency representation,"IEEE ransactions on Signal Processing, vol. 43, no. 10, pp. 2361-2371, 1995.

[21] Zhao Lin-Hai, Wu Jian-Ping, Ran Yi-Kui, "Fault diagnosis for track circuit using AOK-TFRs and AGA," Control Engineering Practice, vol. 20, no. 12, pp. 1270-1280, 2012.

[22] Choi, H., Williams, W., "Improved time-frequency representation of multicomponent signals using exponential kernels,"IEEE Trans. Signal Process, vol. 37, no. 6, pp. 862-871, 1989.

[23] A. Dliou, et al,"Abnormal ECG signals analysis using non parametric time-frequency techniques," The Arabian Journal for Science and Engineering Journal, vol. 39, no. 2, pp 913-921, 2014.

[24] R. Latif, M. Laaboubi, A. Dliou, "Acoustic experimental signal analysis using an adaptive neuro-fuzzy inference system and different time-frequency techniques," Internatinal Review on Computers and Software (IRECOS), vol. 8, no. 1, pp. 313-326, 2013.

[25] S. Elouaham, R. Latif, A. Dliou, M. Laaboubi, F. M. R. Maoulainine, "Biomedical signals analysis using the empirical mode decomposition and parametric and non parametric time-frequency techniques,"International Journal on Information Technology (IREIT), vol. 1,no. 1, pp. 1-10, 2013.

[26] Latif, R.; Laaboubi, M.; Aassif, E.; Maze, G., "Détermination de l'épaisseur d'un tube élastique à partir de l'analyse temps-fréquence de Wigner-Ville,J. Acta-Acustica, vol. 95, no. 5, pp. 843-848, 2009.

[27] S. Elouaham, R.Latif, B.Nassiri, A. Dliou, M. Laaboubi, F. Maoulainine, "Analysis Electroencephalogram signals using ANFIS and Periodogram techniques,"International Review on Computers and Software (IRECOS.), vol. 8, n.12. pp 2959-2966, 2013.

[28] S. Elouaham, R. Latif, A. Dliou, F. M. R. Maoulainine,"Periodogramand ensemble empirica mode decomposition analysis of electromyography processing,"International Journal of Computer Applications, vol. 65, no.2, pp. 33-40, 2013. 